\title{
OPTICAL IN-Fiber BRAgg Grating SENSOR SYSTEMS FOR MEDical APPLiCaTions
}

\author{
Y. J. Rao, ${ }^{\dagger}$ D. J. Webb,${ }^{\dagger}$ D. A. Jackson, ${ }^{\dagger}$ L. Zhang, ${ }^{\dagger}$ and I. Bennion ${ }^{*}$ \\ ${ }^{\dagger}$ University of Kent, Physics Laboratory, Applied Optics Group, Canterbury, Kent CT2 7NR, United \\ Kingdom; ${ }^{\ddagger}$ Aston University, Department of Electronic Engineering, Photonics Research Group, \\ Birmingham B4 7ET, United Kingdom \\ (Paper JBO/IB-003 received Jul. 17, 1997; revised manuscript received Oct. 20, 1997; accepted for publication Oct. 21, 1997.)
}

\begin{abstract}
Two in-fiber Bragg grating (FBG) temperature sensor systems for medical applications are demonstrated: (1) an FBG flow-directed thermodilution catheter based on interferometric detection of wavelength shift that is used for cardiac monitoring; and (2) an FBG sensor system with a tunable Fabry-Perot filter for in vivo temperature profiling in nuclear magnetic resonance (NMR) machines. Preliminary results show that the FBG sensor is in good agreement with electrical sensors that are widely used in practice. A field test shows that the FBG sensor system is suitable for in situ temperature profiling in NMR machines for medical applications. (C) 1998 Society of Photo-Optical Instrumentation Engineers. [S1083-3668(98)00801-6]
\end{abstract}

Keywords fiber-optic sensors, interferometry, in-fiber Bragg gratings, temperature sensors, cardiac monitoring, NMR imaging, microwaves.

\section{INTRODUCTION}

For medical temperature measurements, small, reliable, and inexpensive thermocouple and thermistor devices have been commercially available for many years and are still widely used. However, because these sensors are electrically active, they are not appropriate for a number of medical applications, in particular, in high magnetic fields associated with nuclear magnetic resonance (NMR) machines in which temperature profiles in the human body must be obtained for diagnosis purposes. Fiberoptic sensors can overcome this problem because they are dielectric and hence virtually immune to electromagnetic interference. A range of miniature fiber-optic medical temperature sensors have been reported in recent years and a small number have been successfully commercialized. ${ }^{1-3}$ Generally speaking, these sensors are all point sensors, which can only provide temperature readings over a small volume in the body rather than temperature distribution within the body. Although passive multiplexing of these point sensors is possible, it is difficult to achieve in practice because of limitations on the probe size.

In-fiber Bragg grating (FBG) sensors ${ }^{4-5}$ have received considerable interest in recent years and have been demonstrated for a wide range of applications, from civil engineering, ${ }^{6}$ mines, ${ }^{7}$ and aerospace composites ${ }^{8}$ to medical monitoring. ${ }^{9}$ Compared with the point fiber-optic sensors, FBG sensors have a number of distinguishing advantages: (1) signal processing is simple because the

Address all correspondence to Y. J. Rao. Tel: 441227 764000, Ext 3772; Fax: 441227 827558; E-mail: y.rao@ukc.ac.uk information is directly obtained by detecting the wavelength shift induced by the measurand; (2) FBGs can be written directly into the fiber without changing the fiber diameter $(125 \mu \mathrm{m})$, making them well suited for use in the human body during in vivo temperature profiling; (3) they could be mass produced at low cost, making them potentially competitive with traditional electrical sensors; (4) they can be multiplexed easily with wavelengthdivision-multiplexing (WDM), making quasidistributed sensing feasible in practice. FBG sensing systems can be expected to be cost-effective when the number of sensors that can be multiplexed is large. These features make FBG sensors ideal for temperature profiling in the human body. In this paper, we describe two FBG sensor systems for use in measuring the heart's efficiency and in monitoring temperature distribution in NMR machines for medical applications.

\section{FBG-BASED FLOW-DIRECTED THERMODILUTION CATHETER}

Measurement of the heart's efficiency plays a key role in cardiac monitoring. Since 1970, doctors have injected patients with cold saline solution to measure the blood output of the heart. ${ }^{10} \mathrm{~A}$ flowdirected thermodilution catheter is inserted into the right atrium of the heart. The catheter allows the saline solution to be injected directly into the heart and enables measurement of the temperature of the blood in the pulmonary artery. By combining temperature readings with pulse rate, doctors can determine how much blood the heart pumps. How- 


\section{Conventional Flow-Directed Thermodilution Catheter}

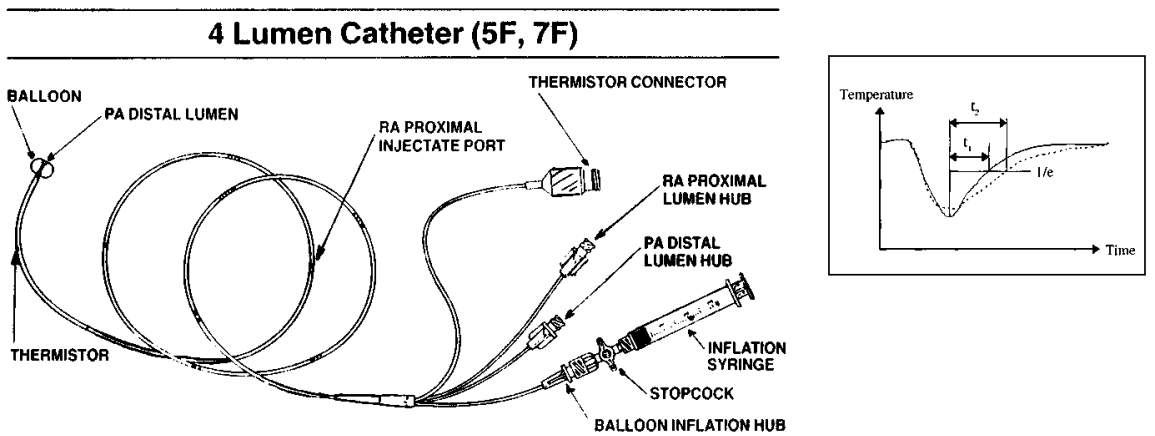

Fig. 1 Schematic diagram of a typical flow-directed thermodilution catheter.

ever, because these sensors are electrically active, they are not appropriate for use in a number of medical applications, in particular, in the high magnetic fields associated with NMR machines.

\subsection{CATHETER BASED ON AN ELECTRICAL SENSOR}

A typical flow-directed thermodilution catheter (Model 250/401/050, Portex Ltd., England) is shown in Figure 1. When the heart rate is known, the time delay $t_{1}$ in the temperature profile shown in the inset of Figure 1 can be used to determine the amount of blood pumped by the heart. A thermistor is used to obtain the temperature profile. If the efficiency of a patient's heart is poor (this may be caused by some blockages in blood vessels), the time delay will become $t_{2}$ where $t_{2}$ becomes larger than $t_{1}$ as the amount of blood the heart can pump during a defined time interval is reduced. The amplitude of the temperature curve is also decreased because the cold solution moves slowly and warms gradually. The temperature distribution measured by the thermistor is affected by: (1) the temperature of the solution; (2) the volume of the solution; (3) the speed of injection; and (4) the size of the catheter. Therefore, careful calibration is normally required. The thermodilution cardiac outputs have a typical reproducibility of $5 \%$.

\subsection{OPTICAL THEORY OF IN-FIBER BRAGG GRATINGS}

An FBG is written into a segment of a Ge-doped single-mode fiber in which a periodic modulation of the core refractive index is formed by exposure to a spatial pattern of ultraviolet light in the region of 244 to $248 \mathrm{~nm}$. This fabrication process is based on the photorefractive effect in the germania oxygen-vacancy defect band, which was observed in Ge-doped optical fibers by Hill et al. ${ }^{11}$ The lengths of FBGs are normally within the region of 1 to $\sim 20 \mathrm{~mm}$ and grating reflectivities can approach $\sim 100 \%$. When the FBG is illuminated by a broadband light source, a set of beams reflected from a set of partially reflecting planes formed by the periodic core index modulation interfere with each other. The interference is destructive unless each beam is in phase with all the others. According to Bragg's law which gives this condition, only one wavelength, i.e., the Bragg wavelength $\lambda_{B}$, is selected. It is given by

$$
\lambda_{B}=2 n \Lambda
$$

where $n$ is the effective core index of refraction and $\Lambda$ is the period of the index modulation.

For a temperature change of $\Delta T$, the corresponding wavelength shift $\Delta \lambda_{B T}$ is given by

$$
\Delta \lambda_{B T}=\lambda_{B}(1+\xi) \Delta T
$$

where $\xi$ is the fiber thermo-optic coefficient. For a silica fiber, the wavelength-temperature sensitivities of $800 \mathrm{~nm}$ and $1.55 \mu \mathrm{m}$ FBGs have been measured with values of $\sim 6.8 \mathrm{pm} /{ }^{\circ} \mathrm{C}$ and $\sim 13 \mathrm{pm} /{ }^{\circ} \mathrm{C}$, respectively. 5

\subsection{FBG-BASED CATHETER}

The schematic diagram of the FBG-based catheter system is shown in Figure 2. Light from a pigtailed temperature-stabilized superluminescent diode (SLD) with a bandwidth of $\sim 18.5 \mathrm{~nm}$ (818 to 836.5 $\mathrm{nm})$, supplied by Superlum Ltd. (Moscow), was launched into an unbalanced scanning interferometer (SI). The optical power output from the singlemode fiber was measured as $\sim 1 \mathrm{~mW}$. The nominal Bragg wavelengths and reflectivities of both the sensing FBG and the reference FBG were $\sim 827.5 \mathrm{~nm}$ and $\sim 90 \%$ with a spectral bandwidth of $\sim 0.2 \mathrm{~nm}$. The SI was a bulk Michelson interferometer acting as a tunable filter. The piezoelectric element in the SI was modulated at frequencies of 300 $\mathrm{Hz}$ with a ramp (serrodyne) function that provides a phase carrier. ${ }^{12}$ The reference FBG is placed in the output arm of fiber coupler 1 while the sensing FBG is arranged in the output arm of coupler 2. By detecting the differential phase between the sensing FBG and the reference FBG located in a well- 


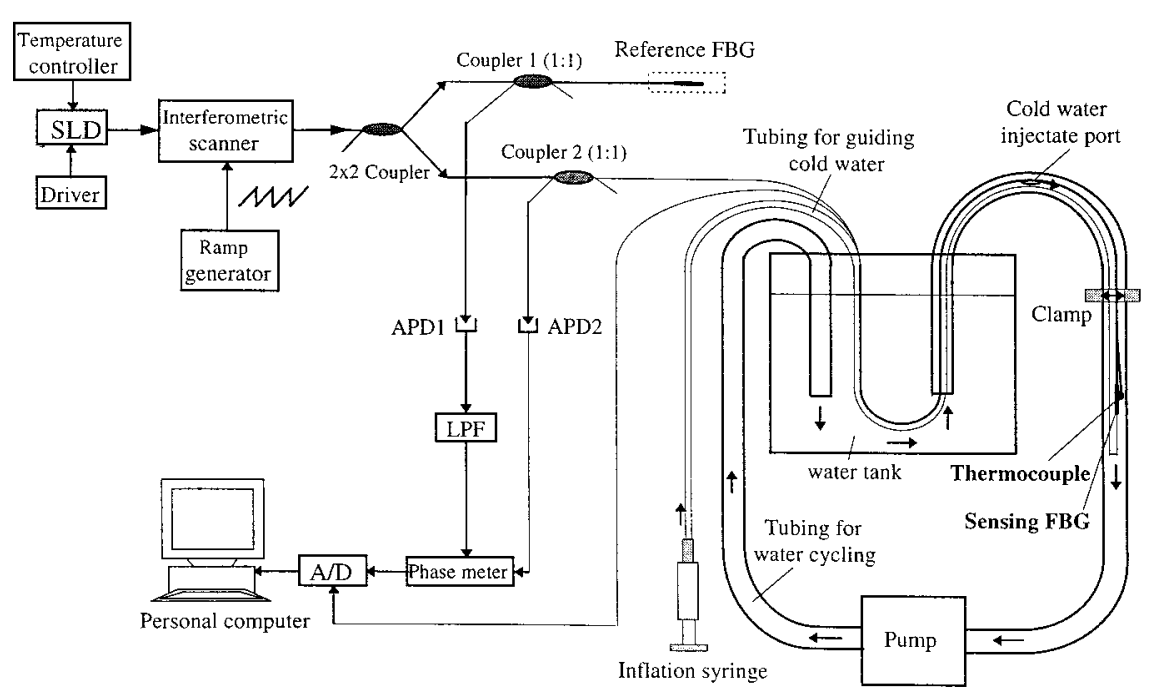

Fig. 2 Schematic diagram of an FBG-based flow-directed thermodilution catheter system; SLD, superluminescent diode; APD1 and APD2, avalanche photodiodes; LPF, low-pass filter; A/D, analog-to-digital converter.

controlled environment, the thermal drifts of the SI can be effectively eliminated. ${ }^{13}$ The differential phase is measured by low-pass filtering at the fundamental frequency of the serrodyne signal and comparing the relative phase of the sinusoidal outputs corresponding to the sensing FBG and the reference FBG with a phase meter (here it is a lock-in amplifier). The normalized interferometric signal of the sensing FBG at avalanche photodetector APD2 can be expressed by

$$
I / I_{0}=\left[1+V \cos \left(\omega t+\Delta \Phi_{B}(t)\right],\right.
$$

where $I_{0}$ is the intensity of the incident light, $V$ is the visibility of the interference signals, and $\omega$ is the serrodyne modulation frequency in the SI. The temperature-induced change in the reflected wavelength from the sensing FBG produces an optical phase change, $\Delta \Phi_{B}(t)$, given by:

$$
\Delta \Phi_{B}(t)=-\frac{2 \pi \Delta L}{\lambda_{B}^{2}} \Delta \lambda_{B}(t)=-\frac{2 \pi \Delta L}{\lambda_{B}} \xi_{g} \Delta T(t),
$$

where $\Delta T(t)$ is the variation in temperature applied to the sensing FBG and $\xi_{g}$ is the normalized FBG wavelength shift to temperature sensitivity. Hence, the optical phase sensitivity in response to temperature, $\Delta \Phi_{B} / \Delta T$, is directly proportional to the optical path difference (OPD) of the SI. However, the OPD is constrained by the coherence length, which is determined by the bandwidth of the FBG used. The OPD of the SI was set as $\sim 2 \mathrm{~mm}$ (which is equal to a free spectral range of $0.35 \mathrm{~nm}$ ) in this work. By measuring $\Delta \Phi_{B}$ with a pseudoheterodyne processing scheme, ${ }^{12}$ the temperature change can be determined.

A test rig was set up to simulate blood flow in the heart and is shown in Figure 2. A peristaltic pump designed for medical purposes was used to simulate the heart pump. The pump rate can be continuously varied between 60 and $120 \mathrm{rpm}$ by changing the dc voltage applied to the motor inside the pump. Warm water $\left(37\right.$ to $\left.40^{\circ} \mathrm{C}\right)$ was put into a water tank and then cold water at room temperature was injected into the tubing via the Portex catheter shown in Figure 1. In order to compare the results optically and electrically, a thermocouple located at the same position as the sensing FBG was used as a temperature reference. The results were recorded by a personal computer (PC) via a 12-bit analog-todigital converter.

\subsection{EXPERIMENTAL RESULTS}

The phase output of the FBG sensor was first calibrated against the output of the thermocouple. The coefficient of the response obtained was $\sim 8 \mathrm{deg} /{ }^{\circ} \mathrm{C}$. The resolution of the FBG sensor system was measured as $\sim 0.2^{\circ} \mathrm{C}$ over a bandwidth of $10 \mathrm{~Hz}$. Two experiments were carried out with the system mentioned earlier. The first one was to obtain the relationship between the pump rate and the time delay. The results are shown in Figure 3. Both the FBG sensor and the thermocouple match well except that there is an offset. This systematic offset may be caused by the fact that the FBG with a length of $4 \mathrm{~mm}$ is much longer than the thermocouple with a diameter of $\sim 0.5 \mathrm{~mm}$ so that the temperature profile obtained by the FBG sensor is further smoothed. This could be an advantage of using the FBG sensor because the influence of any turbulence in blood flow could be averaged and a more accurate measurement of the time delay could be achieved. The second experiment was to simulate the change of blood flow due to a change in the size of the blood vessel when the pump rate is kept constant. For this purpose, a clamp was used to 


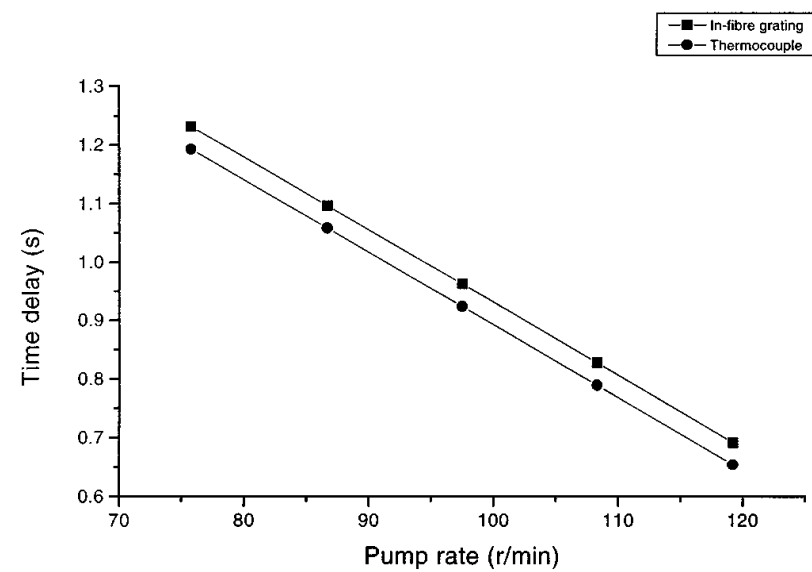

Fig. 3 Experimental results of time delay against pump rate.

squeeze the tubing as shown in Figure 2. The results for both unclamped and clamped cases with a pump rate of $108 \mathrm{rpm}$ are shown in Figures 4(a) and 4(b). It can be seen that for both the FBG sensor and the thermocouple, the time delays have changed from $\sim 0.9 \mathrm{~s}$ to $\sim 1.1 \mathrm{~s}$ due to the partial clamping, which corresponds to a relative change of $\sim 10 \%$. The FBG used for these experiments was a standard unit. Recent research has shown that special FBGs can be produced to give greater sensitivity. Also, wavelength-division-multiplexing has been combined with interferometric detection recently ${ }^{14}$ and hence quasi-distributed measurement could be achieved for monitoring blood flow along the blood vessel.

\section{PROTOTYPE FBG SENSOR SYSTEM FOR USE IN NMR MACHINES}

\subsection{SENSING SYSTEM}

Although the sensor system with interferometric detection can achieve fast measurement with high sensitivity, if it is used for quasi-distributed measurement, WDM is required. Here we describe a simple sensing system that can achieve wavelength detection and multiplexing simultaneously for static measurement of temperature rather than the dynamic measurement mentioned earlier. The schematic diagram of the FBG sensor system developed is shown in Figure 5. This system is similar to that reported by A. D. Kersey ${ }^{15}$ except that there are some differences in operating wavelengths and post-signal processing. Light from the SLD was launched into a tunable-fiber Fabry-Perot filter (FPF), supplied by Micro Optics Inc., U.S., rather than the Michelson interferometer. The FPF, used for both wavelength-shift detection and WDM of the FBG sensors, has a free spectral range (FSR) of $40 \mathrm{~nm}$ and a finesse of 271. The thermal drift of the FPF can be eliminated by measuring the difference between the reference FBG and the sensing FBGs. The sensing FBG array has a range from $\sim 821$ to

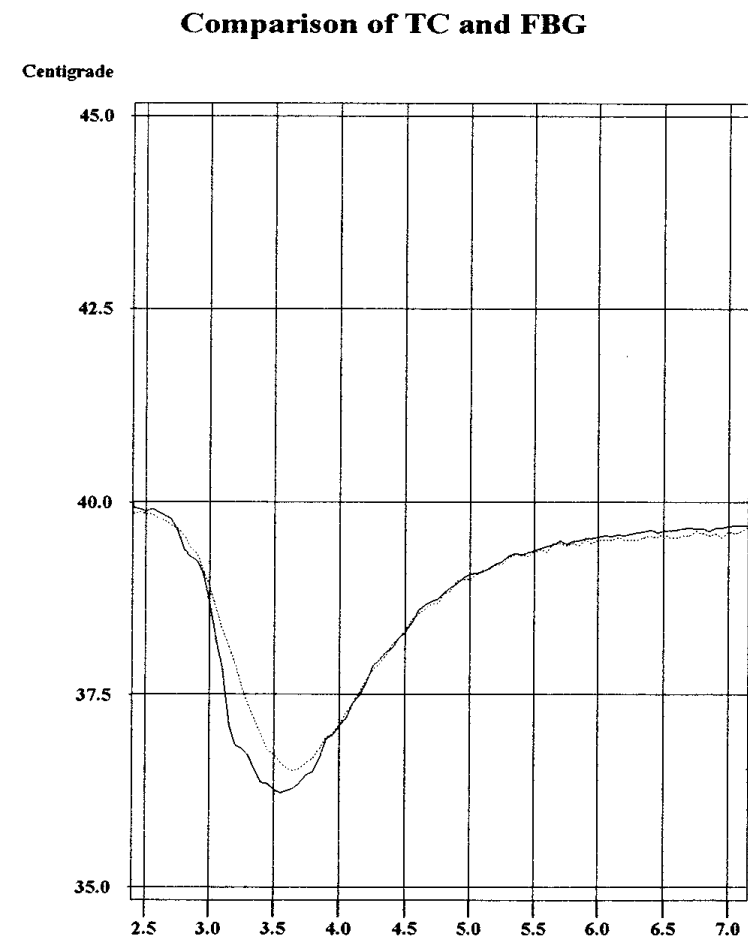

(a)

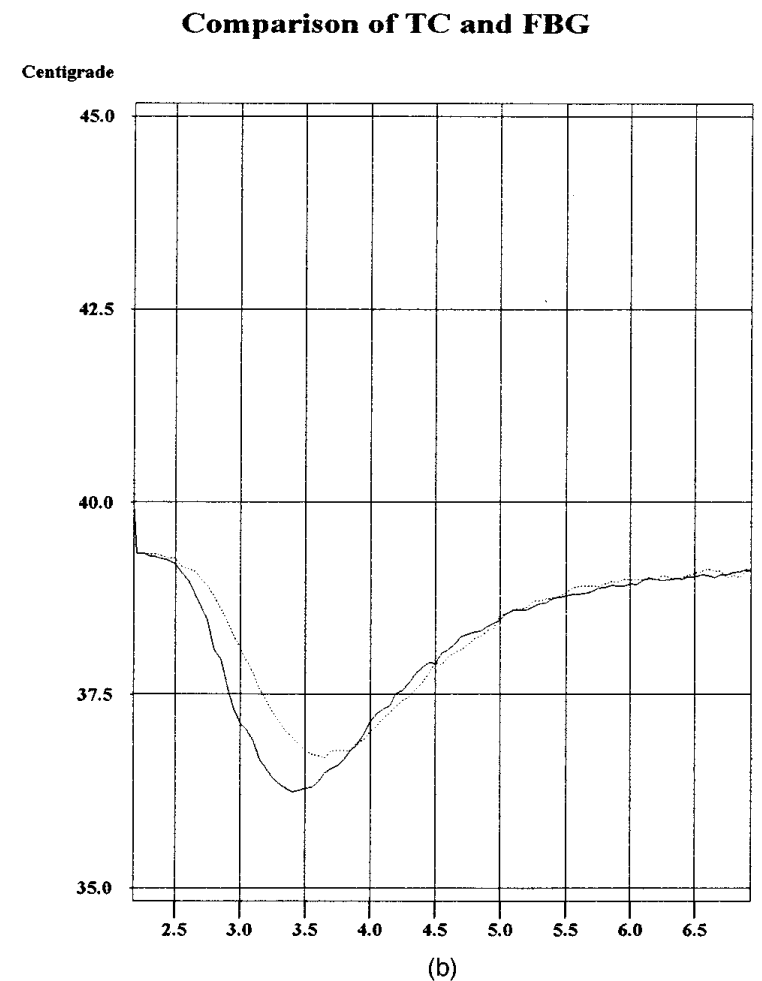

Fig. 4 Experimental results of simulation for blood-vessel blocking (a) without clamping of the tubing and (b) with clamping of the tubing. Solid line, thermocouple; dotted line, in-fiber grating.

$\sim 829 \mathrm{~nm}$, with reflectivities of 50 to $70 \%$. A linear $40-\mathrm{Hz}$ ramp generated by a 16-bit digital-to-analog converter was used to scan the FPF. For an FSR of $40 \mathrm{~nm}$, the 16-bit ramp should give a wavelength- 


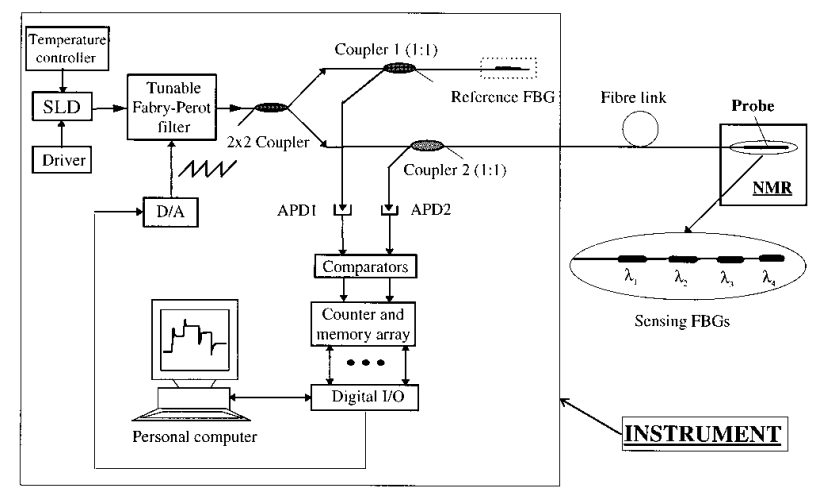

Fig. 5 Schematic diagram of FBG temperature sensor system with an FP tunable filter.

shift resolution of $1.2 \mathrm{pm}$, or an equivalent temperature resolution of $0.2^{\circ} \mathrm{C}$ for $800-n m$ FBGs. The ramp and the detected signals for both the reference FBG and the sensing FBGs are shown in Figure 6. A specific software program was developed to synchronize the ramp and the counters. The central wavelengths of the reference FBG and each sensing FBG were determined by taking the average of two counts corresponding to the leading and falling edge positions of the scanned waveform via a threshold comparator. The difference between the reference FBG and each sensing FBG was averaged for 16 times to reduce noise. This gives a measurement time of $0.4 \mathrm{~s}$, which meets the requirement for

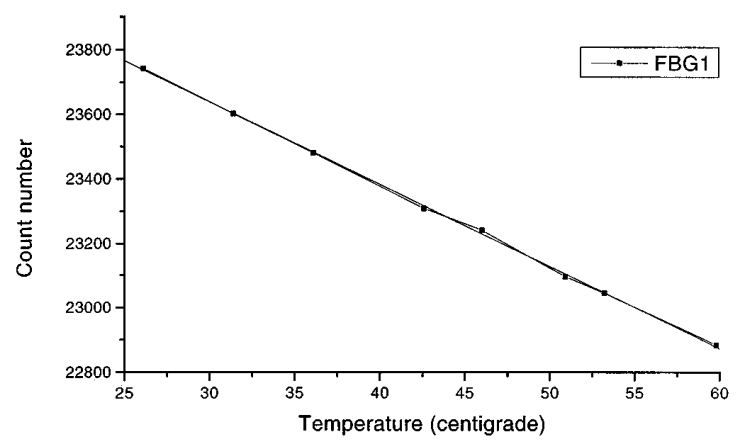

(a)

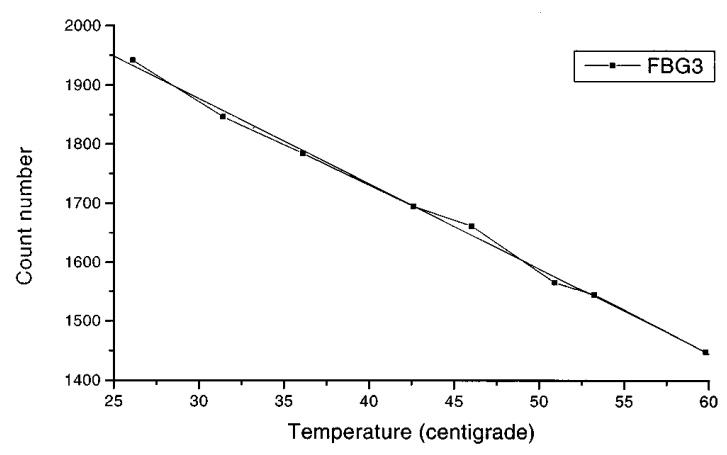

(c)

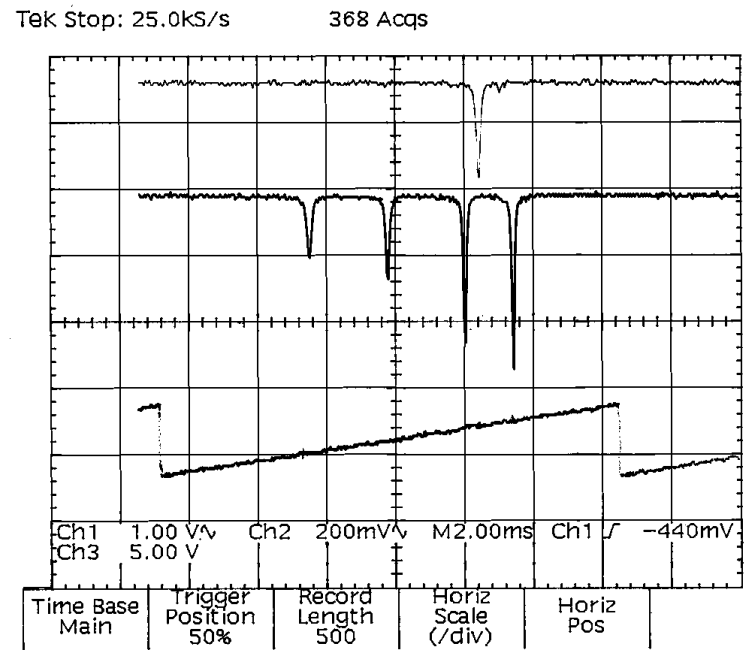

Fig. 6 Waveforms of the linear ramp and detected signals.

medical applications (typically $1 \mathrm{~s}$ ). The counts obtained from ten counters were sent into ten memory chips and then read in the PC via a digital input/ output interface card, respectively. The probe with four sensing FBGs was placed inside an NMR machine with a high magnetic field of $\sim 4.7$ teslas. In situ measurement was achieved by using a fiber line of $\sim 25 \mathrm{~m}$ to link the instrument and the NMR machine.

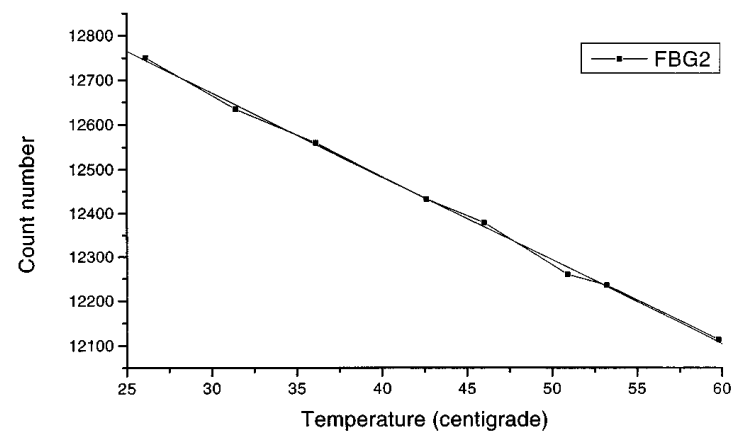

(b)

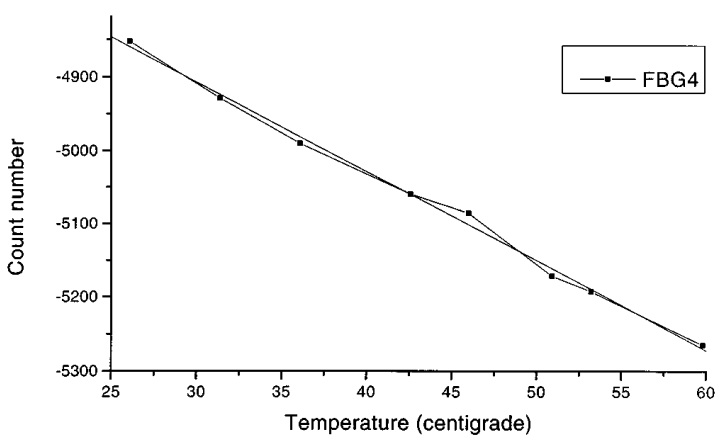

(d)

Fig. 7 Experimental results of sensing FBGs against thermocouple: (a) $\lambda_{1}=821 \mathrm{~nm}$, (b) $\lambda_{2}=824 \mathrm{~nm}$, (c) $\lambda_{3}=827 \mathrm{~nm}$, and (d) $\lambda_{4}=829 \mathrm{~nm}$. 


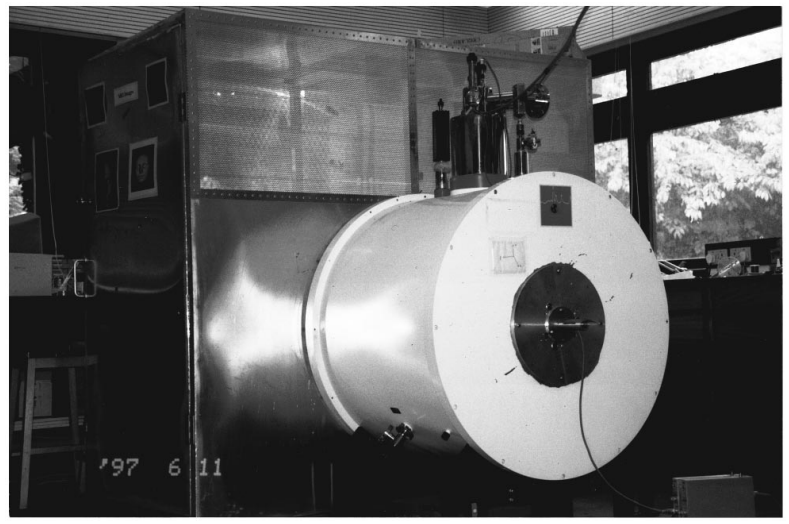

Fig. 8 Photograph of the NMR machine used.

\subsection{EXPERIMENTAL RESULTS}

In order to calibrate the optical system, a thermocouple located at the same position as the sensing FBGs was used as a temperature reference before the sensing FBGs were put into the NMR machine. The results of the count number against temperature are shown in Figures 7(a) to 7(d), which provide the scale factors for the sensing FBGs. It is seen that an accuracy of $\sim \pm 0.8^{\circ} \mathrm{C}$ has been achieved. The accuracy is mainly restricted by the nonlinearity of the piezoelectrical tranducer (PZT) in the FPF. This could be improved by modeling the nonlinearity and then using a nonlinear ramp to drive the PZT. The temperature resolution of this system was obtained by placing the sensing FBGs in a water container at room temperature and then inserting the container in the NMR machine shown in Figure 8. The results are shown in Figure 9 and it can be seen that for all the FBGs used, the resolution was $\sim \pm 0.2^{\circ} \mathrm{C}$. By further increasing the averaging times, the resolution was improved by a factor of 2 , i.e., $\sim \pm 0.1^{\circ} \mathrm{C}$, but the measurement time was longer $(\sim 1.6 \mathrm{~s})$. Real-time measurement was demonstrated by adding warm water and then ice to the container. The experimental results are shown in Figure 10. All the FBG sensors worked well and the

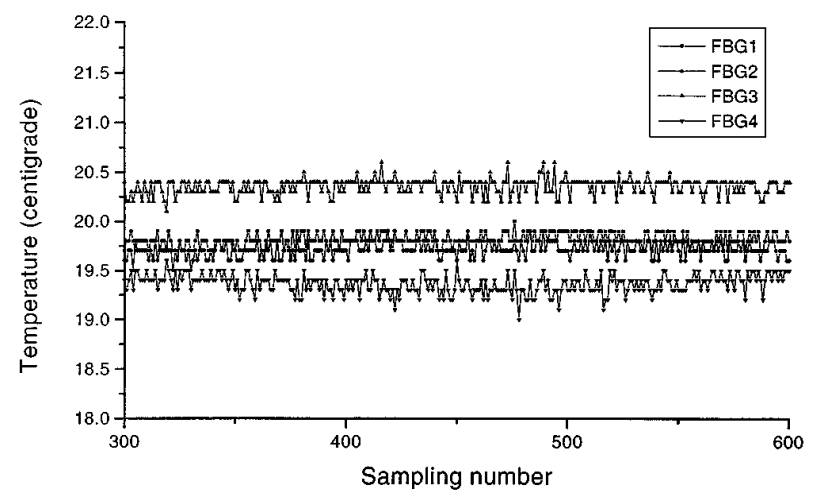

Fig. 9 Noise distribution of the sensing FBGs.

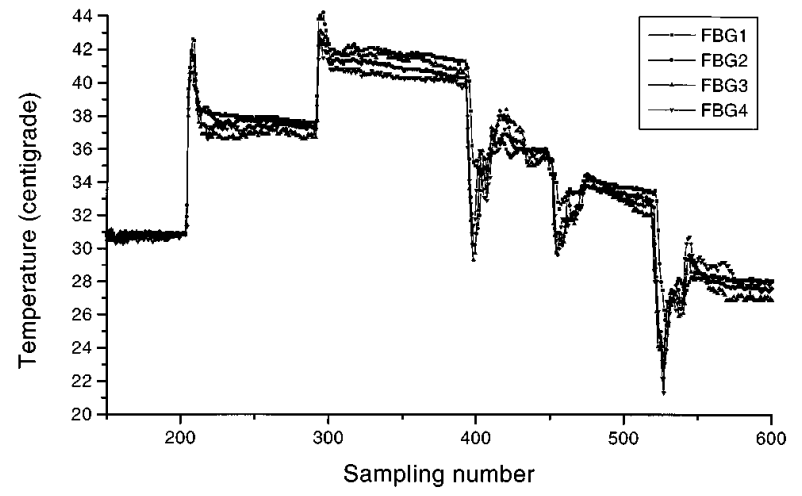

Fig. 10 Experimental results of temperature measurement with FBGs operated in an NMR machine.

transitions caused by suddenly changing the temperature of the water as readily seen.

\section{CONCLUSION}

In summary, we have demonstrated two FBG temperature sensor systems for medical applications. For the FBG flow-directed thermodilution catheter for use in cardiac monitoring, preliminary results show that the FBG sensor is in good agreement with the electrical sensors that are widely used in practice. It was found that because the FBG is longer than electrical sensors, more accurate measurement could be achieved due to smoothing of temperature profiles. It is anticipated that this FBG sensor system could be used for in vivo applications in the near future.

For the prototype FBG sensor system for remote temperature measurement in an NMR machine, a resolution of $\sim \pm 0.2^{\circ} \mathrm{C}$ and an accuracy of $\sim \pm 0.8^{\circ} \mathrm{C}$ have been achieved; these could be further improved. Preliminary results obtained from the field test show that the FBG sensor is well suited for temperature profiling in NMR machines. It is also anticipated that after further engineering, this potentially low-cost, portable, and robust system could be used for in vivo tests and other scientific applications, such as monitoring the temperature of samples analyzed by NMR.

\section{Acknowledgment}

This work was supported by the Wellcome Trust (Project 043154/Z/94/ZIMP/RF/PK). The authors would like to thank the NMR group at the University of Kent for permission to use their NMR machine.

\section{REFERENCES}

1. M. H. Sun, K. A. Wickersheim, and J. Kim, "Fibre-optic temperature sensors in the medical setting," Proc. SPIE 1967, 15-21 (1989).

2. R. A. Wolthuis, G. L. Mitchell, E. Saaski, J. C. Harti, and A. Afromowitz, "Development of medical pressure and temperature sensors employing optical spectrum modulation," IEEE Trans. Biomed. Eng. 38, 974-981 (1991). 
3. Y. J. Rao and D. A. Jackson, "Recent progress in fibre optic low-coherence interferometry," Meas. Sci. Technol. 7, 981999 (1996).

4. W. W. Morey, G. Meltz, and W. H. Glenn, "Fibre optic Bragg grating sensors," Proc. SPIE 1169, 98-107 (1989).

5. Y. J. Rao, "In-Fibre Bragg Grating Sensors," Meas. Sci. Technol. 8, 355-375 (1997).

6. R. M. Measures, A. T. Alavie, R. Maaskant, M. Ohn, S. Karr, and S. Huang, "Bragg grating structural sensing system for bridge monitoring," Proc. SPIE 2294, 53-60 (1994).

7. P. Ferdinand, O. Ferragu, J. L. Lechien, B. Lescop, V. Marty, V. S. Rougeault, G. Pierre, C. Renouf, B. Jarret, G. Kotrotsios, V. Neuman, Y. Depeursings, J. B. Michel, M. V. Uffelen, Y. Verbandt, M. R. H. Voet, and D. Toscano, "Mine operating accurate stability control with optical fibre sensing and Bragg grating technology: the BRITE-EURAM STABILOS project," Proc. SPIE 2360, 162-166 (1994).

8. P. D. Foote, I. J. Read, T. J. Slack, A. Ball, P. Jackson, Y. J. Rao, D. A. Jackson, I. Bennion, and L. Zhang, "Optical fibre Bragg sensors systems: a basis for aircraft health and usage monitoring," submitted to J. Smart Mater. Struct.

9. Y. J. Rao, D. J. Webb, D. A. Jackson, L. Zhang, and I. Bennion, "In-fibre Bragg grating temperature sensor system for medical applications," J. Lightwave Technol. 15, 779-785 (1997).

10. J. S. Forrester et al. "Thermodilution cardiac output determination with a single flow-directed catheter," Am. Heart J. 83, 306-311 (1972).

11. K. O. Hill, F. Fujii, D. C. Johnson, and B. S. Kawasaki, "Photosensitivity on optical fibre waveguides: application to reflection filter fabrication," Appl. Phys. Lett. 32, 647-649 (1978).

12. D. A. Jackson, A. D. Kersey, and M. Corke, "Pseudoheterodyne detection scheme for optical interferometers," Electron. Lett. 18, 1081-1082 (1982).

13. A. D. Kersey, T. A. Berkoff, and W. W. Morey, "Fibre optic Bragg grating strain sensor with drift-compensated highresolution interferometric wavelength shift detection," Opt. Lett. 18, 72-74 (1993).

14. Y. J. Rao, D. J. Webb, D. A. Jackson, L. Zhang, and I. Bennion, "A novel high-resolution, wavelength-divisionmultiplexed in-fibre Bragg grating sensor system," Electron. Lett. 32, 924-926 (1996).

15. A. D. Kersey, T. A. Berkoff, and W. W. Morey, "Multiplexed fibre Bragg grating strain-sensor system with a fibre FabryPerot wavelength filter," Opt. Lett. 18, 1370-1372 (1993). 